\title{
Technetium and the third dredge up in AGB stars
}

\section{Bulge stars ${ }^{\star}$}

\author{
S. Uttenthaler ${ }^{1,2}$, J. Hron ${ }^{2}$, T. Lebzelter ${ }^{2}$, M. Busso ${ }^{3}$, M. Schultheis ${ }^{4}$, and H. U. Käufl ${ }^{1}$
}

1 European Southern Observatory, Karl Schwarzschild Straße 2, 85748 Garching near Munich, Germany e-mail: [suttenth; hukaufl]@eso.org

2 Department of Astronomy, University of Vienna, Türkenschanzstraße 17, 1180 Vienna, Austria

e-mail: [hron;lebzelter]@astro.univie.ac.at

3 Department of Physics, University of Perugia, via A. Pascoli 1, 06123 Perugia, Italy e-mail: busso@fisica.unipg.it

${ }^{4}$ Observatoire de Besançon, 41bis, avenue de l'Observatoire, BP 1615, 25010 Besançon Cedex, France e-mail: mathias@obs-besancon.fr

Received 20 April 2006 / Accepted 12 October 2006

\section{ABSTRACT}

\begin{abstract}
Context. Technetium (Tc) is an unstable element synthesised via the s-process in deep layers of asymptotic giant branch (AGB) stars, making it a reliable indicator of both recent s-process activity and third dredge-up.

Aims. We searched for Tc in a sample of bright oxygen-rich asymptotic giant branch (AGB) stars located in the outer galactic bulge. We test theoretical predictions of the luminosity limit for the onset of third dredge-up.

Methods. Using high-resolution optical spectra obtained with the UVES spectrograph at ESO's VLT, we searched for resonance lines of neutral Tc in the blue spectral region of our sample stars. These measurements allow us to improve the procedure of classification of stars with respect to their Tc content by using flux ratios. Synthetic spectra based on MARCS atmospheric models are presented and compared to the observed spectra around three lines of Tc. Bolometric magnitudes are calculated based on the near-infrared photometry of the objects.

Results. Among the sample of 27 long-period bulge variables, four were found to definitely contain Tc in their atmospheres. The luminosity of the Tc-rich stars agrees with predictions from AGB evolutionary models on the minimum luminosity at the time when third dredge-up sets in. However, AGB evolutionary models and a bulge consisting of a single old population cannot be brought into agreement. This probably means that a younger population is present in the bulge, as suggested by various authors, that contains the Tc-rich stars identified here.
\end{abstract}

Key words. stars: late-type - stars: AGB and post-AGB - stars: evolution

\section{Introduction}

The asymptotic giant branch (AGB) phase is an important step in the late evolution of low and intermediate mass $\left(1-8 M_{\odot}\right)$ stars. In the most luminous part of the AGB, the behaviour of a star is characterised by the so-called thermal pulses (TP), thermal instabilities of the He shell accompanied by changes in luminosity, temperature, period, and internal structure (Busso et al. 1999; Herwig 2005). Between the repeated events of explosive He-burning, heavy elements can be produced via the "slow neutron capture process" (s-process, see e.g. Wallerstein et al. 1997) in the region between the hydrogen- and the helium-burning shells. The processed material is then brought to the stellar surface by the convective envelope that temporarily extends to these very deep layers. This mixing event is called the third dredge-up (3DUP) and is the cause of the eventual metamorphosis of an oxygen-rich $\mathrm{M}$-star into a carbon-rich $\mathrm{C}$-star.

In recent years, considerable progress has been made with regard to models for the 3DUP and nucleosynthesis on the

* Based on observations at the Very Large Telescope of the European Southern Observatory, Cerro Paranal/Chile under Program 65.L-0317(A, B). thermally pulsing AGB (TP-AGB, Busso et al. 1999; Lugaro et al. 2003, and references therein). The different evolution models agree qualitatively in the sense that the 3DUP is more efficient for more massive convective envelopes (e.g. Straniero et al. 1997) and for lower metallicities (e.g. Straniero et al. 2003). However, the quantitative results are still model-dependent (Lattanzio 2002; Lugaro et al. 2003), and grids of new models covering a wider range of stellar parameters are scarce. In general, observed abundances of s-process elements agree with the model predictions, at least qualitatively. For instance, the metallicity dependence of the 3DUP is supported by observations (Busso et al. 2001; Abia et al. 2002), although observations not compatible with the models have to be mentioned here (Deroo et al. 2005; Masseron et al. 2006). A first attempt to directly check the conditions for the onset of 3DUP observationally has been made by Lebzelter \& Hron (1999). Important constraints on the minimum (core) mass (and hence luminosity) for 3DUP and its efficiency also come from the observed luminosity function of carbon stars in the LMC (Costa \& Frogel 1996) and in the Galaxy (Guandalini et al. 2006), together with synthetic stellar evolution calculations (Groenewegen \& de Jong 1993; Marigo et al. 1996). 
Table 1. Basic characteristics of the targets in our sample.

\begin{tabular}{|c|c|c|c|c|c|c|c|}
\hline Name & RA & $\overline{\text { Dec }}$ & Period [d] & $J_{0}[\mathrm{mag}]$ & $K_{0}[\mathrm{mag}]$ & $\mathrm{RV}\left[\mathrm{km} \mathrm{s}^{-1}\right]$ & Tc? \\
\hline M45 & 181248.5 & -331927 & 271.02 & 8.19 & 6.69 & +19.8 & no \\
\hline M100 & $\begin{array}{lll}18 & 13 & 38.5\end{array}$ & -364003 & 298.7 & 7.65 & 6.37 & -37.3 & no \\
\hline M143 & 181413.6 & -323658 & 204.19 & 9.32 & 8.00 & +17.6 & no \\
\hline M195 & 181507.7 & -330922 & 216.59 & 8.88 & 7.61 & -142.8 & no \\
\hline M277 & 181615.4 & -314249 & 263.23 & 8.55 & 7.16 & -34.5 & no \\
\hline M315 & 181645.0 & -324001 & 326.8 & 8.02 & 6.58 & -61.9 & no \\
\hline M331 & 181657.6 & -320629 & 311.07 & 8.17 & 6.64 & -31.8 & no \\
\hline M626 & 182132.5 & -360735 & 298.48 & 8.44 & 7.19 & -67.7 & yes \\
\hline M794 & 182428.0 & -323051 & 303.54 & 7.48 & 6.07 & -51.0 & no \\
\hline M1147 & 183306.2 & -362227 & 395.63 & 7.23 & 5.76 & +1.0 & yes \\
\hline M1179 & 183354.7 & -350119 & 274.51 & 8.56 & 7.20 & +61.8 & no \\
\hline M1287 & 183644.5 & -322543 & 312.5 & 8.03 & 6.67 & +229.4 & no \\
\hline M1313 & 183735.5 & -341227 & 378.7 & 8.42 & 6.79 & +14.1 & no \\
\hline M1347 & 183845.7 & -343328 & 426.6 & 7.47 & 5.99 & +60.5 & yes \\
\hline S70 & 181305.6 & -322341 & 166.52 & 8.56 & 7.19 & -77.2 & no \\
\hline S328 & 181656.2 & -335324 & 161.3 & 9.01 & 7.78 & -117.9 & no \\
\hline S639 & 182142.3 & -350308 & 167.3 & 8.74 & 7.46 & -88.7 & no \\
\hline S719 & 182318.4 & -360505 & 279.77 & 7.90 & 6.61 & +69.4 & no \\
\hline S942 & 182809.0 & -363126 & 338.0 & 7.86 & 6.54 & -77.8 & yes \\
\hline S1002 & 182922.8 & -314416 & 194.23 & 8.41 & 7.11 & +33.0 & no \\
\hline S1008 & 182934.1 & -340827 & 232.14 & 8.00 & 6.73 & -39.0 & no \\
\hline S1059 & 183042.8 & -360036 & 144.1 & 8.91 & 7.68 & +18.0 & no \\
\hline S1176 & 183343.8 & -312735 & 184.1 & 8.38 & 6.99 & +56.1 & no \\
\hline S1204 & 183438.8 & -340008 & 197.0 & 8.67 & 7.32 & +236.5 & no \\
\hline S1470 & 184231.7 & -355928 & 184.08 & 8.51 & 7.33 & -37.7 & no \\
\hline S1517 & 182719.1 & -320633 & 188.8 & 9.03 & 7.71 & +80.2 & no \\
\hline S1991 & 181531.4 & -315840 & 124.7 & 9.28 & 8.06 & -102.1 & no \\
\hline
\end{tabular}

Technetium (Tc) is among the elements produced by the s-process, and it has no stable isotopes. The isotope of Tc with the longest half-life time produced via the s-process is ${ }^{99} \mathrm{Tc}$ with $\tau_{1 / 2}=2.1 \times 10^{5}$ years. In the following discussion, we always refer to the isotope ${ }^{99} \mathrm{Tc}$ when we discuss Tc. The short half-life time makes Tc a reliable indicator of the 3DUP, because any Tc we see in a star must have been produced during its previous evolution on the TP-AGB. Tc should be detectable at the surface after only a few thermal pulses (Goriely \& Mowlavi 2000). It should be noted at this point that the absence of Tc does not necessarily mean the absence of TPs but rather the absence of 3DUP for several TPs. This could be caused by an initial mass on the TP-AGB that is too low or by a mass-loss rate at the end of the AGB-evolution that is too high (e.g., Busso et al. 1992).

A number of studies have been published on observations of Tc in spectra of late type stars, starting from the first observation by Merrill (1952), through investigations by Dominy \& Wallerstein (1986), Wallerstein \& Dominy (1988), Smith \& Lambert (1988, 1990), Vanture et al. (1991), to studies on S-stars (Van Eck \& Jorissen 1999) and C-stars (Barnbaum \& Morris 1993). Important studies of Tc in large samples of longperiod variables have been conducted by Little-Marenin \& Little (1979), Little et al. (1987), and Lebzelter \& Hron (1999, 2003).

In the first paper of this series (Lebzelter \& Hron 2003, hereafter called Paper I), the Tc content of a sample of luminosityselected galactic field AGB stars was studied. A significant number of stars above the luminosity limit for 3DUP, indicated by mixing models, were found to not show Tc in their spectra. This can be explained by the fact that the second important parameter for 3DUP is the mass of the envelope. It is suspected that the absence of Tc in a significant fraction of long-period Miras is due to a reduction of the envelope mass below the critical limit by mass loss and/or due to a low initial mass of the star. Due to the uncertainties in distance (based on Hipparcos parallaxes) of field stars, no definite conclusions could be drawn.

A sample of targets with more accurate distances is required to improve the situation. Given the current accuracy of the distance measurements of AGB stars and the low flux in the blue spectral region of these stars, the only available targets for such studies can be found in the galactic bulge. The distance to the bulge $(8 \mathrm{kpc})$ is known rather accurately, and the depth of the bulge is low enough, at least in the outer parts, to have a fairly low depth-induced scatter in brightness $(+0.5 /-0.96$, Schultheis et al. 1998). Using ESO's VLT, exposure times are short enough to execute observations of a statistically relevant sample in a reasonable time. Additionally, the bulge population is expected to be more homogeneous than the disk population, because the widely absent $\mathrm{C}$-stars indicate that high-mass stars are no longer present in the bulge. We therefore chose to observe bright AGB stars in the Palomar Groningen field No. 3.

It should be noted, however, that AGB variables in the bulge do not have the same average properties as their disk counterparts, especially the semiregular variables (SRVs). These differences might be explained by a different age-metallicity relation and a different pulsation mode for the bulge SRVs compared to the field SRVs. For details we refer to Schultheis et al. (1998), while the present paper focuses on dredge-up indicators rather than pulsation properties.

The paper is structured in the following way. The sample selection is presented in Sect. 2, possible foreground and background contamination is discussed in Sect. 3. In Sect. 4 the UVES observations are presented, while Sects. 5-7 deal with the basic characteristics of the sample stars summarised in Table 1, the detection of Tc, and the discussion of the data, respectively. Conclusions can be found in Sect. 8. 


\section{Sample selection}

The selection of the sample was limited to oxygen-rich longperiod variables in the Palomar Groningen field No. 3 (PG3), which is centred $10^{\circ}$ south of the galactic centre and covers an area of $6.5 \times 6.5$ on the sky. It is located in the outer bulge where interstellar extinction is rather low and the depth of the bulge is small. The PG3 field has been studied extensively in the past (Wesselink 1987; Blommaert 1992; Ng 1994; Schultheis 1998; Schultheis et al. 1998) and periods are known for a considerable number of variable stars.

To avoid foreground or background objects, we constructed a period $K$-magnitude diagram based on near-infrared photometry acquired at the ESO 1-m telescope at La Silla (see Schultheis et al. 1998, and references therein) and additional data from DENIS (Epchtein et al. 1997) for some stars. The periods were taken from Wesselink (1987). A range of \pm 1 m 0 mag around the period $K$-magnitude relation for the SgrI field from Glass et al. (1995) was allowed for the potential targets to account for the depth of the bulge and the intrinsic scatter in brightness (partly only single-epoch measurements available). Stars outside this range were considered to be in the foreground or background. The targets were chosen to be brighter than the RGB tip (8.2 mag in $K_{0}$ at the bulge distance, see Tiede et al. 1995; Omont et al. 1999) to sample the AGB upwards. The distribution was chosen to be about equal between semiregular and Mira variables. This procedure resulted in a preliminary target list for the WFI observations.

\section{Fore- and background contamination}

Foreground confusion is a serious problem when observing in the direction of the bulge. The density of stars drops fairly rapidly behind the bulge, and a star would have to be extremely bright to fall on the period-luminosity relation. Thus background contamination can be considered as low, and we can restrict the discussion to foreground contamination.

Since long-period variables obey period-luminosity relation(s), their distance can be inferred from their position in such a diagram with a certain accuracy. However, the membership of single stars in the disk cannot be excluded if no kinematic information (proper motion) is available. Close-by M dwarfs in the galactic disk and stars ascending the RGB can be excluded from our sample since neither fulfils the variability criteria of longperiod variables.

To estimate the possible foreground contamination, we used the Besançon model of population synthesis (Robin et al. 2003). As a representative field, we calculated the population of the central square degree of the PG3 field located at $b=-10^{\circ}$ and $l=0^{\circ}$ using the observed photometric ranges (apparent $K$ magnitude, $(J-K)_{0}$ colour) as criteria (the extinction towards the PG3 field is rather low, see Sect. 5). No criteria for the pulsation could be included, but stars with too low a bolometric magnitude were treated as non-variable. The result indicates foreground AGB contamination at a level of $2.4 \%$, which gives on average less than 1 star in a sample of 27 (the final number of objects). Thus, we are confident we only have bulge stars in our sample.

\section{Observations}

From the preliminary target list, 27 objects were selected for the UVES observations. Details of this high-resolution optical spectrograph can be found in Dekker et al. (2000). Pulsation phases of the targets were roughly estimated from preceding
$B$-band Wide Field Imager (WFI) observations obtained at the ESO/MPG $2.2 \mathrm{~m}$ telescope during two runs in April and May 2000. These measurements were used for the final sample selection in an attempt to only observe targets with their maximum brightness close to the time of the UVES observations. The final object selection was also strongly driven by the apparent target brightness at the time of the UVES observations. Table 1 lists some basic characteristics of the final targets. In addition to the bulge objects, a few field AGB stars that have partly been checked for their content of Tc previously (see Paper I and references therein) were observed.

The observations with UVES at ESO's Very Large Telescope (VLT), Cerro Paranal/Chile, were carried out between July 6 and July 9, 2000. The setting was chosen in order to cover the blue (central wavelength $4370 \AA$ ) and red (central wavelength $8600 \AA$ ) arm of the spectrometer simultaneously. The observed wavelength ranges thus were approximately 3770-4900 A (blue arm), 6670-8470 A (red lower arm), and 8650-10500 A (red upper arm). With the blue arm, several resonance lines of neutral Tc were covered, among them the lines at 4238.19, 4262.27, and $4297.06 \AA$ (wavelength in air, hereafter referred to as the "classical" lines). The slit width of the spectrometer was set to 0 . 7 , which results in a resolution of $\lambda / \Delta \lambda \cong 50000$. The spectra were taken in the $1 \times 2$ binning mode. Cumulative exposure times ranged from 600 to $7200 \mathrm{~s}$, with $3600 \mathrm{~s}$ as a typical value. All stars observed during the first night were observed again on the second or third night because the exposure times chosen the first night were not sufficient.

The spectra were reduced with the ESO-provided pipeline written in MIDAS, version 2.1.0. For the spectra in the blue arm, "optimal" extraction was used during the reduction process, whereas "average" extraction was used for the red arm. This procedure is recommended by the pipeline manual to optimise the signal-to-noise ratio (SNR) of the spectra. In optimal extraction, the pipeline performs a Gaussian fit to the signal profile in the spatial direction; the SNR is then computed from the deviation of the profile from this fit.

The achieved SNR of the UVES spectra varies strongly with wavelength. For the regions around the Tc lines, the SNR provided by the UVES pipeline lies between 5 and 40 with the majority of the stars having an SNR of 15 or better. The four stars with Tc have an SNR of around 30 (M626, M1347, S942) and 5 (M1147), respectively. We demonstrate below that it is possible to decide whether Tc is present at such a low SNR.

\section{Sample characteristics}

Table 1 lists some basic characteristics of the observed targets. Column 1 lists the stellar identifier, adopted from Wesselink (1987) which codes the variability type: M stands for Mira variable, $\mathrm{S}$ for semiregular variable. The J2000 coordinates in Cols. 2 and 3 are the (rounded) positions as found in the 2MASS catalogue (Cutri et al. 2003).

The periods in Col. 4 are taken from Wesselink (1987). For two stars (S942 and M315), they give a low quality flag for the period determination. Using their period measurement (176 and $173.6 \mathrm{~d}$, respectively), these two objects would be placed outside the range of the bulge in the period $K$-magnitude diagram. We searched the literature and found a published period of $338 \mathrm{~d}$ for S942 based on photometric data (Plaut 1971). Note also that this star was classified as a Mira variable by Plaut (1971). For M315 a light curve from the MACHO survey could be extracted (as a matter of fact, this star is the only one in our sample covered 
by the MACHO fields). Using the program PERIOD04 (Lenz \& Breger 2004) a robust period of $326.8 \mathrm{~d}$ was determined.

The $J_{0}$ and $K_{0}$ magnitudes, found in Cols. 5 and 6 of Table 1, are the de-reddened mean values of the ESO, DENIS, and 2MASS measurements. We thus have two to five measurements in both bands for our stars, with two measurements for only four of the objects (partly due to DENIS values with a bad quality flag). The de-reddening was performed using the linear relation for the reddening in the $B_{J}$ band as a function of galactic latitude as given in Schultheis et al. (1998). To translate this into the extinction in the $J$ and $K$ bands, we used the relation $R=A_{V} / E(B-V)$ with $R=3.2$ and the reddening law of Glass \& Schultheis (2003). This results in an extinction of around $0.1 \mathrm{mag}$ in $\mathrm{J}$ and a few $0.01 \mathrm{mag}$ in $\mathrm{K}$.

To cross check our approach we also determined the extinction in $\mathrm{K}$ for the respective object positions using the RGB fitting method, taking the RGB of 47 Tuc as reference (following a suggestion by Messineo et al. 2005; Dutra et al. 2003). We extracted all objects $4^{\prime}$ around the target position from the 2MASS catalogue and constructed a $K_{\mathrm{S}}$ versus $J-K_{\mathrm{S}}$ diagram. The computed shift relative to the reference RGB resulted in a small (few 0.01 ) or even negative extinction value. This proves that there is no strong, patchy absorption in the direction of any one of the sample stars, but it also proves that this method is too insensitive in the outer bulge to reliably determine the extinction.

The radial velocities in Col. 7 of Table 1 were determined from the UVES spectra using a cross correlation technique with a synthetic spectrum as a template. For each of the blue, red lower, and red upper arm wavelength ranges a region of around $50 \AA$ was used for the correlation. All three regions were chosen to avoid very broad absorption and emission lines. For the respective region in the red lower arm, the $\mathrm{TiO}$ band head at $7054 \AA$ was covered, since this sharp feature leads to a very accurate velocity measurement. The scatter of the derived radial velocities from the three wavelength regions is of the order of $1 \mathrm{~km} \mathrm{~s}^{-1}$.

Taking a search radius of $30^{\prime \prime}$, a corresponding IRAS source could be found for eight of the sample stars (see also Blommaert 1992). The identification was checked using the 2MASS $K$-images. Where available, the flux from the IRAS Faint Source Catalogue (Moshir et al. 1989) was taken; otherwise, the IRAS Catalogue of Point Sources (Joint IRAS Science W.G. 1994) entry was taken, since the relative flux uncertainty quoted is lower in the former. The IRAS-colours $K_{0}-[12]$ and [12]-[25] are summarised in Table 2. Note that colours were not calculated using flux ratios but rather zero-point magnitudes for each filter. These are 28.3 Jy in [12] and 6.73 Jy in [25] (Joint IRAS Science W.G. 1988). The errors are based on the relative flux uncertainty for the [12] and [25] flux as given in the respective catalogue and on the error-bar in $\mathrm{K}$ used in Fig. 3. The respective colour value is quoted in brackets if a flux with quality flag lower than 2 is involved, and no error is given for these cases.

The two stars with Tc in this small sample are the ones with the longest pulsation period. They have the lowest values for their IRAS [12]-[25] colour, but are otherwise inconspicuous. From Fig. 21 of Whitelock et al. (1994) and using the $K_{0}-$ [12] colour, we can estimate the mass loss of the IRAS sources in our sample to be in the range $-6.8 \lesssim \log \left(\dot{M} / M_{\odot} \mathrm{yr}^{-1}\right) \lesssim$ -6.0 . Selecting optically-bright targets for spectroscopy naturally avoids highly obscured (i.e., high mass loss) objects. We therefore assume that the "Tc yes" stars do not have a
Table 2. IRAS colours of the objects that could be identified either in the IRAS Faint Source Catalogue (Moshir et al. 1989) or in the IRAS Point Source Catalogue (Joint IRAS Science W.G. 1994).

\begin{tabular}{llll}
\hline \hline Name & IRAS ident. & $K_{0}-[12]$ & {$[12]-[25]$} \\
\hline M100 & $18102-3640$ & $2.49 \pm 0.26$ & $1.63 \pm 0.23$ \\
M315 & $18134-3241$ & $2.14 \pm 0.14$ & $(1.78)$ \\
M331 & $18136-3207$ & $2.13 \pm 0.23$ & $(1.92)$ \\
M1147 & $18297-3624$ & $2.36 \pm 0.10$ & $0.64 \pm 0.15$ \\
M1179 & $18305-3503$ & $3.32 \pm 0.30$ & $0.92 \pm 0.39$ \\
M1313 & $18342-3415$ & $2.92 \pm 0.33$ & $0.93 \pm 0.26$ \\
M1347 & $18354-3436$ & $2.11 \pm 0.16$ & $0.77 \pm 0.18$ \\
S1204 & $18313-3402$ & $2.51 \pm 0.12$ & $(1.96)$ \\
\hline
\end{tabular}

considerably higher mass loss and intrinsic reddening than do the other stars in our sample.

\section{Tc detection}

To determine whether or not a star shows Tc, we first inspected the spectra visually around the classical Tc lines together with synthetic spectra (Fig. 1). The synthetic spectra are based on the MARCS (Model Atmospheres in a Radiative Convective Scheme) hydrostatic atmospheric models from Gustafsson et al. (1975) with improvements introduced by Jørgensen et al. (1992) and with spherical radiative transfer routines from Nordlund (1984). The spectral synthesis was recently improved by Gorfer (2005), and atomic line wavelengths were taken from the VALD data base (Kupka et al. 1999) ${ }^{1}$. For Tc, the $g f$-values of Bozman et al. (1968) were used. The synthetic spectra were convolved with a Gaussian to reduce them to a resolution of 50000 , matching the resolution of the observed spectra. No additional macroturbulence was assumed. This assumption was checked by determining the FWHM of a Gaussian fit to a selection of a few strong, seemingly unblended lines of $\mathrm{Fe}, \mathrm{V}, \mathrm{Ti}$, and $\mathrm{Cr}$ in one of the observed spectra with high SNR and the generic synthetic spectrum (see below). The assumption of zero macroturbulence turned out to be acceptable, because the line-widths were never broader by more than $1.2 \mathrm{~km} \mathrm{~s}^{-1}$ in the observed spectrum compared to the model spectrum.

On visual inspection, three stars were identified displaying Tc lines. In Fig. 1 we show sections of the observed spectra around the classical Tc lines of these stars and of one star without Tc. In the lowest panel, two synthetic spectra calculated with and without Tc, respectively, are shown. The synthetic spectra are based on a MARCS atmospheric model with $T_{\text {eff }}=3400 \mathrm{~K},[\mathrm{Fe} / \mathrm{H}]=-0.5, \log (g)=0.0$, one solar mass, solar $\mathrm{C} / \mathrm{O}$ ratio, and a microturbulent velocity of $\xi=2.5 \mathrm{~km} \mathrm{~s}^{-1}$. The atmospheric parameters are not meant as a fit to the real objects but rather are the result of an "educated guess". The spectrum with Tc was calculated assuming a Tc abundance of $\log (\mathrm{A}(\mathrm{Tc}) / \mathrm{A}(\mathrm{H}))+12.0=0.0$. According to Schatz (1983), the equilibrium Tc abundance resulting from the s-process is 0.37 on this scale. Admittedly, the model spectra do not fit the observed spectra very well as the lines seem to be much more pronounced in the model spectra than what is observed. Hence, we do not over-plot them on the observed spectra but plot them separately in the lower panel of Fig. 1. The reason for this discrepancy is not entirely clear. Reducing the metallicity for the model spectra calculation by -1.5 dex results in comparable line strengths.

\footnotetext{
1 As it seems not to be documented elsewhere, we note that the wavelengths of VALD are vacuum values below $2000 \AA$, for longer wavelengths they are standard atmosphere values!
} 


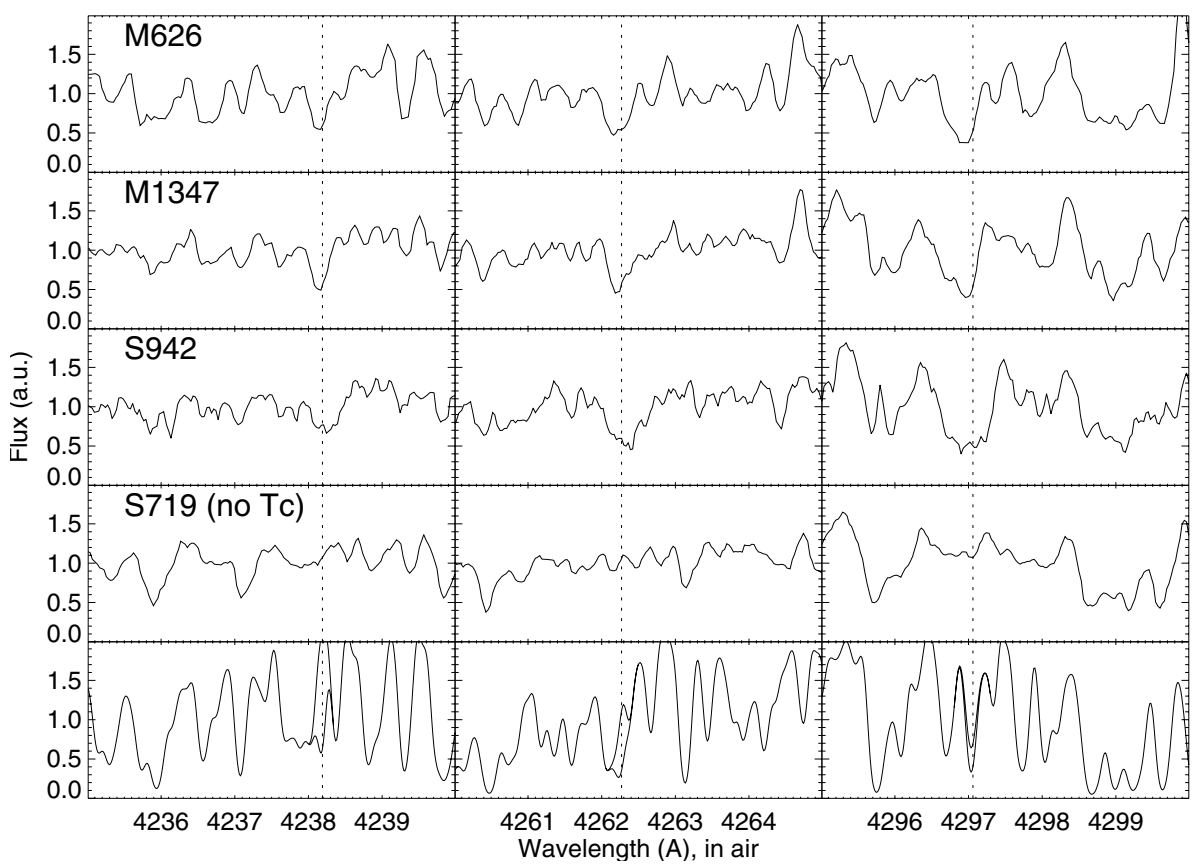

Fig. 1. Regions around the three classical lines of neutral Technetium covered by the UVES spectra. The three upper panels show the stars with definite Tc detection and high signalto-noise ratio, the fourth panel shows a star without Tc. The theoretical positions of the Tc lines are marked by the dotted vertical line. The synthetic spectra with and without Tc in the lower panel are based on a hydrostatic MARCS atmospheric model with $T_{\text {eff }}=$ $3400 \mathrm{~K},[\mathrm{Fe} / \mathrm{H}]=-0.5, \log (g)=0.0$, one solar mass, solar $\mathrm{C} / \mathrm{O}$ ratio, and a microturbulent velocity of $\xi=2.5 \mathrm{~km} \mathrm{~s}^{-1}$. The spectrum with Tc was calculated with a Tc abundance of $\log (\mathrm{A}(\mathrm{Tc}) / \mathrm{A}(\mathrm{H}))+12.0=0.0$. The residual line at the position of the $4297 \AA$ Tc line in the spectrum calculated without Tc is due to chromium. All spectra are normalised so that the mean over the respective plotted region is 1.0 .
Such low metallicity is unrealistic for stars of an inferred age of $3 \mathrm{Gyr}$ and metallicity estimates for the PG3 field (Schultheis et al. 1998) that are slightly above the LMC metallicity. Also, a higher temperature of the sample stars does not serve as an explanation, since in this case no agreement in the strength of the TiO band heads could be found, and the $J-K$ colours are incompatible with a considerably higher temperature. Various authors (e.g. Merrill et al. 1962; Dominy \& Wallerstein 1986) reported on a similar phenomenon observed in field Miras and named - in the absence of a clear physical explanation - "line weakening". This effect is apparently not accounted for in the hydrostatic model atmospheres. Currently, the dynamic model atmospheres of Höfner et al. (2003) are under investigation for their ability to model atomic line strengths in the blue/visual range.

In Fig. 1, the three stars classified as containing Tc by visual inspection clearly show the Tc lines. At the position of the $4297 \AA$ Tc line there appears to also be a somewhat smaller line in the star that was classified as not having Tc (lower panel). This line is caused by chromium (Little-Marenin \& Little 1979). In the three stars identified to show Tc by visual inspection, the Tc lines at 3984.97, 4031.63, 4049.11 and $4095.67 \AA$ identified by Bozman et al. (1968) and listed in the NIST atomic line database (http://physics.nist.gov/ cgi-bin/AtData/lines_form) were also clearly identified, whereas they are absent in the other stars.

For a more quantitative determination of the presence of Tc, we defined flux ratios between the Tc line centre and a pseudocontinuum point close to the line position. This continuum point was selected to be free of any visible absorption features in all observed spectra with good SNR, and in representative synthetic spectra. In a plot of this ratio versus the respective ratio for another Tc line, all stars that were identified to have Tc by visual inspection clearly separate from the other stars. This was done for several pairs of Tc lines always giving the same qualitative result. In Fig. 2 we show this ratio for the lines at 4238 and $4262 \AA$, as these appear to be the strongest and least blended lines. The error bars on the flux ratios were estimated by adding random noise with the magnitude of the inverse SNR that was

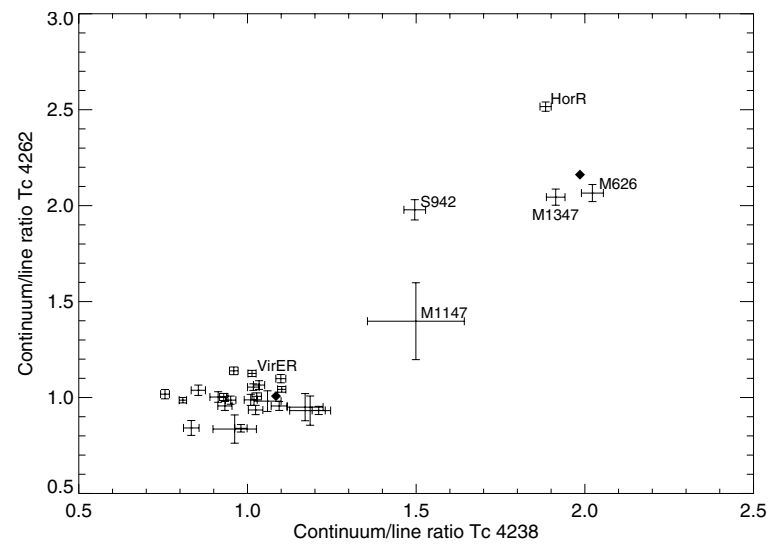

Fig. 2. Continuum-to-line flux ratios for the Tc lines at $4238 \AA$ and $4262 \AA$. For the former, the flux between 4239.4-4239.7 $\AA$ (continuum) was ratio-ed to the flux in the range 4238.0-4238.3 $\AA$ (line), while for the latter the wavelength ranges were 4261.3-4261.5 $\AA$ and 4262.1-4262.3 $\AA$, respectively (see also Fig. 1). Six to ten pixels are typically covered by these ranges. We include the field stars R Hor and ER Vir (analysed in Paper I) for this plot. Also the star M1147 separates quite clearly from the compact group of stars without Tc. This star was not suspected to have Tc from the visual inspection of its spectrum (due to the low SNR). The filled diamond symbols represent the flux ratios derived from the synthetic spectra plotted in Fig. 1.

provided by the pipeline to the observed spectrum. The standard deviation in the flux ratio derived from 100 such realisations of the spectrum gives the error bar. M1147, a star not suspected of showing Tc based on the visual inspection, separates from the compact group of "Tc no" stars as well, although not so obviously. Although this star is the brightest one in the sample in the $K$ band, its flux in the blue spectral region is so low that the SNR per pixel around the Tc lines is as low as 5 after one hour of integration. Also taking the flux ratios of the other identified Tc lines and the error bars from our simulation into account, this star has to be classified as "Tc yes", maybe with a somewhat reduced Tc abundance with respect to the other "Tc yes" stars. For the few other stars with a low SNR, the occurrence of Tc 


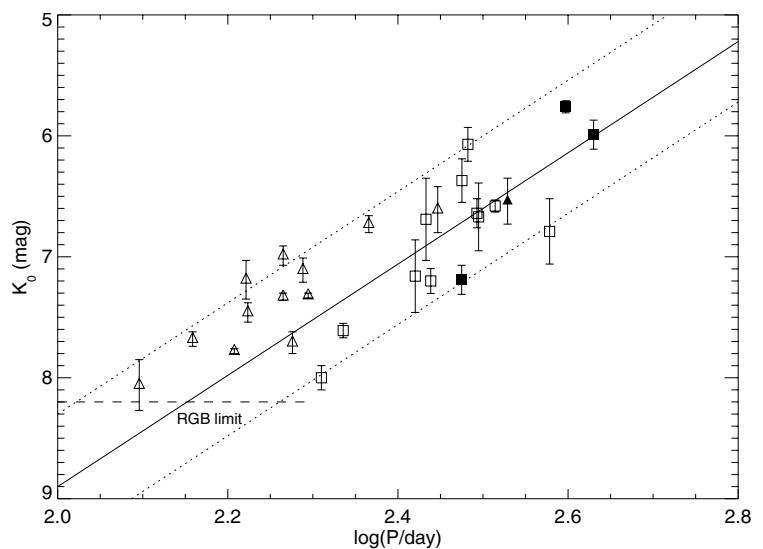

Fig. 3. Period $-K$ magnitude diagram for our sample of long-period variables in the galactic bulge. Squares and triangles represent Mira and semiregular variables, respectively. The filled symbols are stars with a positive Tc detection. The solid line is the period $-K$ relation from Glass $\&$ Schultheis (2003).

can be definitively excluded based on these flux ratios. We also include the flux ratio of the synthetic spectra shown in Fig. 1 in this diagram.

The Tc-rich stars are among the coolest and longest-period objects in our sample; thus, we can exclude the possibility that any noteworthy Tc abundance could have been overlooked in the increasing density of the line forest. On the other hand, Tc has been detected in significantly hotter field stars (e.g. $o^{1}$ Ori, Lebzelter \& Hron 1999). Thus we may safely assume that, within the temperature range of AGB stars, the detection probability is independent of the star's temperature.

Besides Tc, the isotopic ratio ${ }^{12} \mathrm{C} /{ }^{13} \mathrm{C}$ is certainly useful as another indicator of 3DUP. It can be determined from $\mathrm{CO}$ lines present especially in the $K$ band. The advantage of Tc lines as a 3DUP indicator is their complete independence from the star's chemical history due to the radioactive nature of Tc. This certainly is not the case for the ${ }^{12} \mathrm{C} /{ }^{13} \mathrm{C}$ ratio, since it is at least influenced by the initial isotopic composition and by the effects of the first (and, in higher mass stars, the second) dredge-up. Additionally, Tc gives evidence of a recent s-process as well. For comparison, a study on the ${ }^{12} \mathrm{C} /{ }^{13} \mathrm{C}$ ratio in our sample stars and its correlation to the presence of $\mathrm{Tc}$ is under way.

\section{Discussion}

\subsection{Membership in the bulge}

As the basis for the discussion on the bulge membership of the sample stars, we show a period $K$-magnitude diagram in Fig. 3. The error-bar in $K$ is the statistical standard deviation of the mean.

We plot the relation from Glass \& Schultheis (2003, sequence "C") instead of the relation from Glass et al. (1995) that was used for the sample selection. The former is an improved version of the latter. Although Glass \& Schultheis (2003) note that "a very few extra observations could change the slope", the rather steep slope of Glass \& Schultheis (2003), which is based on single-epoch observations in K, fits our multi-epoch data (two to five measurements in $\mathrm{K}$ ) quite well. The dotted lines 0.6 above and 0.5 below sequence " $\mathrm{C}$ " mark the range in magnitude due to the finite depth of the bulge (Schultheis et al. 1998). In Glass \& Schultheis (2003), sequence " $C$ " extends in the range $2.2<\log P<2.7$. In the absence of an alternative relation for the shortest period stars, we plot this over the whole diagram. The dashed line gives the approximate upper limit of the RGB (Tiede et al. 1995; Omont et al. 1999; Zoccali et al. 2003). Using this PL-relation, all stars can be considered to be located in the bulge, within the error bars.

Groenewegen \& Blommaert (2005) published a $\log P-K$ relation for bulge AGB variables based on OGLE light curves, as well as on 2MASS and DENIS data. Since their linear regression to sequence "C" is significantly below the data points for $\log P>2.5$ (see their Fig. 3), we do not use their relation here. Unfortunately, none of our sample stars is covered by the OGLE survey. Even using the relation of Groenewegen \& Blommaert (2005) and adopting the same range in magnitude for bulge stars as before, only one of the Tc-rich stars (M1147) would be placed in the foreground. Thus, the conclusion that AGB stars in the bulge with recent dredge-up are identified is not altered.

Apparently, the SRVs fall mainly above the period $K$-magnitude relation in Fig. 3. As the PG3 SRVs are in the same pulsation mode as the Mira variables (Schultheis et al. 1998), this can be explained by a selection bias towards brighter, i.e. closer, SRVs, as stars on the far side of the bulge may fall below the chosen RGB limit. Detection and non-detection of Tc is marked in this diagram by filled and open symbols, respectively.

The "Tc yes" star with the shortest period is M626 with a period slightly below $300 \mathrm{~d}$. Both stars with the longest period in our sample (M1347 and M1147) show Tc. This compares well with the findings of Paper I where the fraction of "Tc yes" among Mira variables increases above a period of $300 \mathrm{~d}$. Following the variability classification of Plaut (1971), only Miras are found to show Tc in their spectrum (we still keep the SRV symbol for S942 in the figures).

\subsection{Third dredge-up luminosity limit}

To assess theoretical predictions of the minimum luminosity required for 3DUP to occur, we constructed a colour-luminosity diagram shown in the upper panel of Fig. 4. The bolometric magnitudes were calculated from the $K$ brightness and a bolometric correction based on $(J-K)$ using the relation of Kerschbaum et al. (in preparation). This is based on near-infrared and IRAS photometry of a large collection of long-period field variables. Their relation leads to bolometric magnitudes that are $0.08 \mathrm{mag}$ fainter on average than when using the relation of Whitelock et al. (2000). Furthermore, a distance modulus of 14.5 to the bulge was assumed (McNamara et al. 2000).

In Paper I we estimated the minimum luminosity required for 3DUP. This was derived from the luminosity evolution of a $1.5 M_{\odot}$ model at the time when 3DUP sets in (Straniero et al. 1997). At solar metallicity, $1.5 M_{\odot}$ is about the minimum initial mass required for a star to experience 3DUP on the AGB. This minimum luminosity corresponds to a bolometric magnitude of $M_{\text {bol }}=-3$. 9 . As can be seen, all stars with positive Tc detection clearly fall above this line, confirming the theoretically estimated luminosity limit for 3DUP.

The scatter in magnitude of our sample stars around the $\log P-K$ relation may be for various reasons: incomplete lightcurve coverage, depth effects within the bulge, or a scatter in mass and metallicity. For the lower panel of Fig. 4 we assume the scatter to be solely depth-induced (the periods are known with a much higher precision than the $K$-magnitudes). To correct for this scatter, we subtract (or add) the difference between measured $K$ magnitude and the period $K$-magnitude relation of Glass $\&$ Schultheis (2003) from (or to) the bolometric magnitude. In other words, we use the PL-relation to calculate a distance 


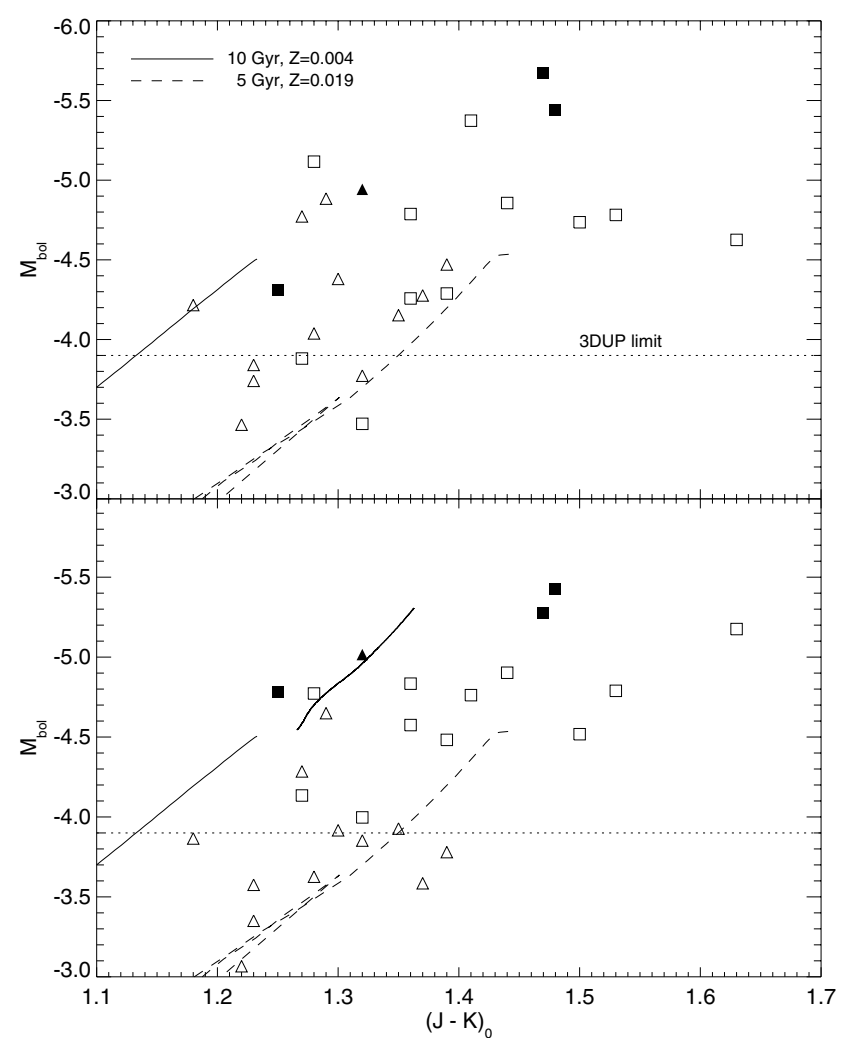

Fig. 4. Colour-luminosity diagram of the sample stars, with symbols the same as in Fig. 3. In the upper panel, the luminosities are plotted as directly derived from the near infrared photometry, whereas in the lower panel the luminosity is corrected for the depth-induced scatter using Fig. 3. The dotted horizontal line marks the minimum luminosity at the stage where 3DUP sets in. Isochrones from Girardi et al. (2000) are also represented in solid and dashed lines (see legend). For both isochrones, the tip of the AGB is well below the observed AGB tip. The thick line in the lower panel is a $1.5 M_{\odot}$ evolutionary model track from Straniero.

modulus for every single star. Applying this correction, the "Tc yes" stars are the brightest objects at a given $(J-K)_{0}$ colour.

\subsection{The mass and age of the Tc stars}

From stellar evolution models (Straniero et al. 2003) one would expect a minimum initial mass limit of $1.4(Z=0.004)$ to $1.5 M_{\odot}$ (solar metallicity) for a star to experience 3DUP. This implies a limiting age of the "Tc yes" stars in our sample of 3 to 4 Gyr.

Various age estimates of the bulge can be found in the literature. Schultheis et al. (1998) give an age range of 5 to $10 \mathrm{Gyr}$ from their study of AGB variables in the PG3 field. Zoccali et al. (2003) obtained colour-magnitude diagrams in the visual and near-IR range and favour a single age of $10 \mathrm{Gyr}$, although an age of $5 \mathrm{Gyr}$ cannot be completely excluded from their analysis (The WFI field studied by Zoccali et al. 2003, does not overlap with the PG3 field, but is situated slightly closer to the galactic plane). Groenewegen \& Blommaert (2005) studied the Mira population in the OGLE bulge fields and derive an age of 1-3 Gyr. Also Zoccali et al. (2003) find a number of stars that are significantly brighter than the estimated RGB tip but do not interpret this as a sign of an intermediate-age population. Studies of the inner part of the bulge (van Loon et al. 2003) and of the galactic bar (Cole $\&$ Weinberg 2002) have found signatures of an intermediate age
(1 to several Gyr) population on top of the main old component. One column figure

An interesting age indicator may come from the period distribution of the Miras in our sample. As argued by Hughes $\&$ Wood (1990), the period distribution of Miras found in the LMC can be understood as a combination of an intermediate and an old population among these variables. Short-period Miras (around $200 \mathrm{~d}$ ) are thought to be older and more metal-poor than their long-period counterparts (Hron 1991). As our sample also includes both short- and long-period Miras, we may suspect that the bulge contains stars of a considerable age range as an analogy to the LMC and the galactic field. Groenewegen \& Blommaert (2005) also conclude from the period distribution of the Mira stars in the OGLE fields that the long-period stars must originate from an intermediate-age population.

In Fig. 4 we include two isochrones from Girardi et al. (2000) with two age-metallicity combinations (see legend). The original $(J-K)$ colours of Girardi et al. (2000) do not reach values above 1.3 and thus do not bracket the observational data properly. This is probably related to the fact that the colours were derived from hydrostatic model atmospheres, while most of the red luminous stars are strongly pulsating objects with dynamic atmospheres. Therefore we chose an observational approach and combined the effective temperatures of Girardi et al. (2000) with a $(J-K)$ vs. $T_{\text {eff }}$ calibration determined from interferometric data and near-infrared photometry of field Mira variables (see Schultheis et al. 1998). With our calibration, the isochrones reach redder $(J-K)$ colours, thus becoming a more realistic description of the observations. But still, the isochrones do not cover the reddest objects, and both parameter sets have their AGB tip at a luminosity fainter by one magnitude than what is observed. The picture is not changed in the second version (lower panel) of the diagram. The extension to red colours illustrates the general difficulty of transforming the effective temperature of a static evolutionary model into colours of a strongly pulsating atmosphere, a systematic investigation of this point is desirable.

The problem of the tip luminosity is discussed in the following. The isochrone of $2.82 \mathrm{Gyr}$ age of Girardi et al. (2000) has an AGB tip luminosity of $M_{\mathrm{bol}}=-4 \mathrm{~m}$. 8 . Taking the luminosity variation during a thermal pulse cycle (see next paragraph) into account thus slightly underestimates the AGB tip luminosity. This implies that models with large overshoot from the envelope, like the ones of Girardi et al. (2000), lead to somewhat lower luminosities than what is observed.

In the lower panel of Fig. 4 (corrected for the depth scatter), we include an evolutionary model track from O. Straniero (priv. comm.). The model used here is similar to the ones presented in Straniero et al. (1997), except that mass loss is taken into account in the current model. The same $(J-K)$ vs. $T_{\text {eff }}$ calibration as for the isochrones was used to include the model track into this diagram. The parameters of the model are as follows: initial mass $1.5 M_{\odot}$, metallicity $Z=0.02$, Helium fraction $Y=0.28$, and Reimers mass-loss parameter $\eta=0.5$ (Reimers 1975). The track covers only the evolution between the next-to-last and the last TP of this model, excluding the short-term luminosity spikes at the TPs themselves (the luminosity in between the preceding TPs is only slightly lower). The track spans about 60000 years. The evolution runs from the lower left end to the upper right end of the track. In other words, the luminosity increases between two TPs while the temperature drops, until the next TP "resets" the star back to low luminosity and a high temperature. The bolometric magnitude varies by 0.75 between two TPs, whereas the temperature varies only slightly between 3176 and $3112 \mathrm{~K}$. 


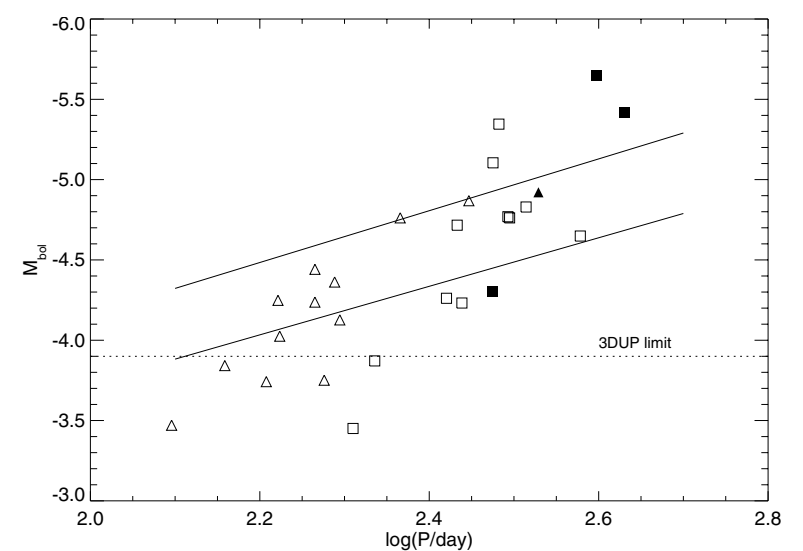

Fig. 5. Bolometric magnitude versus period of the sample stars. Again, the symbols are the same as in the previous figures. The dotted horizontal line marks the minimum bolometric magnitude at the stage where 3DUP sets in. The solid lines are relations for a $1 M_{\odot}$ (lower line) and a $1.5 M_{\odot}$ (upper line) model star pulsating in fundamental mode (Wood \& Sebo 1996).

The luminosities of the evolutionary model track match those of the "Tc yes" stars quite closely, especially the tip luminosity. Only the range in $(J-K)$ of the observed stars is wider than that of the model track, either because the "Tc yes" stars span a wider range in temperature or because of the mentioned problems involved in the $T_{\text {eff }}$ to $(J-K)$ transformation. In light of these results, it is not surprising to find stars which are more luminous than the tip of the Girardi isochrones. All "Tc yes" stars are found there, suggesting that these stars are indeed the most evolved and most massive ones in our sample.

Besides the evolutionary track of Straniero, the interpolation formulae of Straniero et al. (2003) were used for a comparison with the present data set. With these formulae, the luminosity and the temperature at half the time between two successive TPs can be calculated. Very good agreement with the observational data could be found by using the same parameters as for the full model track.

Trusting in the nucleosynthesis and stellar evolution models alone, our results would require that the bulge includes a population of stars with an age around 3 Gyr. On the other hand, if the age estimates of Schultheis et al. (1998) and Zoccali et al. (2003) are correct, then dredge-up would occur at a minimum mass significantly lower than $1.4 M_{\odot}$. This would then imply that the maximum AGB luminosity predicted by the stellar evolution models like the ones from Straniero et al. (2003) is too low.

Finally, in Fig. 5 we present an $M_{\mathrm{bol}}-\log (P)$ diagram of our sample stars. The distribution of the objects is very similar to the one in Fig. 3. The solid lines show the theoretical relations from Wood \& Sebo (1996) for a $1 M_{\odot}$ (lower line) and a $1.5 M_{\odot}$ model (upper line) in fundamental mode pulsation. As for the evolution models, masses around $1.5 M_{\odot}$ are required to fit the brighter, long-period pulsators in the present sample. This supports the solution discussed above that there is a young, more massive population present in the outer bulge and that the predictions of mixing theories are correct.

\section{Conclusions and outlook}

We have presented high-resolution UVES/VLT spectra and NIR photometry of bulge AGB variables, aiming to detect the third dredge-up indicator Technetium. The bulge membership of these stars was discussed using a period $K$-magnitude diagram. In a sample of 27 stars, four were found to have Tc, giving the first direct evidence of recent or ongoing third dredge-up in these stars. For the distinction between "Tc no" and "Tc yes" stars, we compared the observed spectra to synthetic ones around the "classical" Tc lines. A more precise distinction is possible using flux ratios between the Tc line flux and a pseudo-continuum flux. Using this method, even for very low SNR spectra (down to 5), a reliable distinction of this kind can be made.

In a colour-luminosity diagram of the sample stars, all objects with Tc clearly fall above the theoretical third dredgeup limit of $M_{\text {bol }}=-3$.9, in agreement with model predictions. Many stars above the theoretical luminosity limit for third dredge-up do not show Tc. There have been suggestions that Tc could decay (even below the detection limit) if 3DUP does not occur for several TPs (see Busso et al. 1992; and Paper I). One may speculate that the star M1147 has a reduced Tc abundance with respect to the other three "Tc yes" stars, although it is one of the brightest (and most evolved) objects in the present sample. It is possible that this star on the AGB tip has lost so much mass that the envelope is no longer massive enough to drive dredgeup, and Tc has already decayed to a lower abundance level.

The observed AGB tip luminosity is well reproduced by full evolutionary model tracks, assuming an initial mass of $1.5 M_{\odot}$ and a Reimers-parameterised mass loss. Isochrones assuming large overshoot from the convective envelope are found to somewhat underestimate the AGB tip luminosity, although a younger age of the population would reduce this discrepancy.

The disagreement between observed spectra and synthetic spectra based on hydrostatic atmospheric models with respect to atomic line strengths in the blue/visual range is a striking phenomenon found for pulsating AGB variables. In the near future, dynamic models such as those from Höfner et al. (2003), will be tested for their ability to reproduce the observed line strengths.

From the period distribution, the period-luminosity diagram, and the detection of Tc as incontestable indicator for 3DUP, a mass of about $1.5 M_{\odot}$ is required for at least some of the sample stars. This implies an upper age limit of around $3 \mathrm{Gyr}$ for these stars, consistent with other findings of an intermediate age population in the bulge (van Loon et al. 2003; Groenewegen \& Blommaert 2005). In contrast, Zoccali et al. (2003) do not find any signatures of an intermediate age population, but favour a single age of $10 \mathrm{Gyr}$ for the bulge. A solution to this disagreement cannot be given here and must await future work on the galactic bulge.

Acknowledgements. We wish to thank B. Aringer and M. Gorfer for improvements in the spectral synthesis calculation and for inclusion of Tc line data. We also thank M. Messineo (ESO Garching) for inspiring and helpful discussion, and O. Straniero (Teramo, Italy) for providing the AGB evolution models used here.

The constructive comments of the referee (M. Reyniers) were very helpful for improving the paper.

T.L. acknowledges funding by the Austrian Science Fund FWF under project P18171.

This publication makes use of data products from the Two Micron All Sky Survey, which is a joint project of the University of Massachusetts and the Infrared Processing and Analysis Center/California Institute of Technology, funded by the National Aeronautics and Space Administration and the National Science Foundation.

This research has made use of the SIMBAD database, operated at CDS, Strasbourg, France.

\section{References}

Abia, C., Domínguez, I., Gallino, R., et al. 2002, ApJ, 579, 817 Barnbaum, C., \& Morris, M. 1993, BAAS, 182, 46.17 
Blommaert, J. A. D. L. 1992, Ph.D. Thesis, Leiden University, The Netherlands Bozman, W. R., Corliss, C. H., \& Tech, J. L. 1968, Journal of Research Nat. Bur. Stand., 72A, No. 6, 559

Busso, M., Lambert, D. L., Beglio, L., et al. 1992, ApJ, 399, 218

Busso, M., Gallino, R., \& Wasserburg, G. J. 1999, ARA\&A, 37, 239

Busso, M., Gallino, R., Lambert, D. L., Travaglio, C., \& Smith V. V. 2001, ApJ, 557,802

Cole, A. A., \& Weinberg 2002, ApJ, 574, L43

Costa, E., \& Frogel, J. A. 1996, AJ, 112, 2607

Cutri, R., Skrutskie, M., Van Dyk, S., et al. 2003, The Two Micron All Sky Survey Catalogue of Point Sources

Dekker, H., D'Odoricio, S., Kaufer, A., et al. 2000, Proc. SPIE, 4008, 534

Deroo, P., Reyniers, M., van Winckel, H., et al. 2005, A\&A, 438, 987

Dutra, C. M., Santiago, B. X., Bica, E. L. D., \& Barbuy, B. 2003, MNRAS, 338, 253

Dominy, J. F., \& Wallerstein, G. 1986, ApJ, 310, 371

Epchtein, N., de Batz, B., Capoani, L., et al. 1997, ESO Messenger, 87, 27

Girardi, L., Bressan, A., Bertelli, G., \& Chiosi, C. 2000, A\&AS, 141, 371

Glass, I. S., Whithelock, P. A., Catchpole, R. M., \& Feast, M. W. 1995, MNRAS, 273,383

Glass, I. S., \& Schultheis, M. 2003, MNRAS, 345, 39

Gorfer, M., Diploma thesis, University of Vienna, Austria

Goriely, S., \& Mowlavi, N. 2000, A\&A, 362, 599

Groenewegen, M. A. T., \& de Jong, T. 1993, A\&A, 267, 410

Groenewegen, M. A. T., \& Blommaert, J. A. D. L. 2005, A\&A, 443, 143

Guandalini, R., Busso, M., Ciprini, S., et al. 2006, A\&A, 445, 1069

Gustafsson, B., Bell, R. A., Eriksson, K., \& Nordlund, A. 1975, A\&A, 42, 407

Herwig, F. 2005, ARA\&A, 43, 435

Höfner, S., Gautschy-Loidl, R., Aringer, B., \& Jørgensen, U. G. 2003, A\&A, 399, 589

Hron, J. 1991, A\&A, 252, 583

Hughes, S. M. G., \& Wood, P. R. 1990, AJ, 99, 784

Joint IRAS Science Working Group 1988, IRAS Catalogues and Atlases, Volume 1: Explanatory Supplement, NASA RP-1190

Joint IRAS Science Working Group 1994, IRAS catalogue of Point Sources, Version 2.0

Jørgensen, U. G., Johnson, H. R., \& Nordlund, A. 1992, ASPC, 26, 540

Kerschbaum, et al. 2006, in preparation

Kupka, F., Piskunov, N., Ryabchikova, T. A., Stempels, H. C., \& Weiss, W. W. 1999, A\&AS, 138, 119

Lattanzio, J. C. 2002, New Astron. Rev., 46, 469

Lebzelter, T., \& Hron, J. 1999, A\&A, 351, 533
Lebzelter, T., \& Hron, J. 2003, A\&A, 411, 533 (Paper I)

Lenz, P., \& Breger, M. 2004, IAU Symp., 224 (Cambridge University Press), 786

Little, S. J., Little-Marenin, I. R., \& Hagen-Bauer, W. 1987, AJ, 94, 981

Little-Marenin, I., \& Little, S. J. 1979, AJ, 84, 1374

Lugaro, M., Herwig, F., Lattanzio, J. C., Gallino, R., \& Straniero, O. 2003, ApJ, 586,1305

Marigo, P., Bressan, A., \& Chiosi, C. 1996, A\&A, 313, 545

Masseron, T., Van Eck, S., Famaey, B., et al. 2006, A\&A, 455, 1059

McNamara, D. H., Madsen, J. B., Barnes, J., \& Ericksen, B. F. 2000, PASP, 112 , 202

Merrill, P. W. 1952, ApJ, 116, 21

Merrill, P. W., Deutsch, A. J., \& Keenan, P. C. 1962, ApJ, 136, 21

Messineo, M., Habing, H. J., Menten, K. M., et al. 2005, A\&A, 435, 575

Moshir, M., et al. 1989, IRAS Faint Source Survey, Infrared Processing and Analysis Center, California Institute of Technology, Pasadena

Ng, Y. K. 1994, Ph.D. Thesis, Leiden University, The Netherlands

Nordlund, A. 1984, in Methods in Radiative Transfer, ed. W. Kalkofen (Cambridge: Cambridge University Press), 211

Omont, A., et al. 1999, A\&A, 348, 755

Plaut, L. 1971, A\&AS, 4, 75

Reimers, D. 1975, Mem. Soc. Roy. Sci. Lièege, 6th Ser., 8

Robin, A. C., Reylé, C., Derrière, S., \& Picaud, S. 2003, A\&A, 409, 523

Schatz, G. 1983, A\&A, 122, 327

Schultheis, M. 1998, Ph.D. Thesis, University of Vienna, Austria

Schultheis, M., Ng, Y. K., Hron, J., \& Kerschbaum, F. 1998, A\&A, 338, 581

Smith, V. V., \& Lambert, D. L. 1988, ApJ, 333, 219

Smith, V. V., \& Lambert, D. L. 1988, ApJS, 72, 387

Straniero, O., Chieffi, A., Limongi, M., et al. 1997, ApJ, 478, 332

Straniero, O., Domínguez, I., Cristallo, S., \& Gallino, R. 2003, PASA, 20, 389

Tiede, G. P., Frogel, J. A., \& Terndrup, D. M. 1995, AJ, 110, 2788

Van Eck, S., \& Jorissen, A. 1999, A\&A, 345, 127

van Loon, J. T., Gilmore, G. F., Omont, A., et al. 2003, MNRAS, 338, 857

Vanture, A. D., Wallerstein, G., Brown, J. A., \& Bazan, G. 1991, ApJ, 381, 278

Wallerstein, G., \& Dominy, J. F. 1988, ApJ, 330, 937

Wallerstein, G., Iben, I., Parker, P., et al. 1997, Rev. Mod. Phys., 69, 995

Wesselink, Th. J. H. 1987, Ph.D. Thesis, Catholic University of Nijmengen, The Netherlands

Whitelock, P. A., Menzies, J., Feast, M., et al. 1994, MNRAS, 267, 711

Whitelock, P. A., Marang, F., \& Feast, M. W. 2000, MNRAS, 319, 728

Wood, P. R., \& Sebo, K. M. 1996, MNRAS, 282, 958

Zoccali, M., Renzini, A., Ortolani, S., et al. 2003, A\&A, 399, 931 